\title{
Collective electromagnetic relaxation in crystals of molecular magnets
}

\author{
Collin L. Joseph, Carlos Calero, and Eugene M. Chudnovsky \\ Department of Physics and Astronomy, Lehman College, City University of New York \\ 250 Bedford Park Boulevard West, Bronx, New York 10468-1589, U.S.A.
}

(Dated: July 12, 2018)

\begin{abstract}
We study the magnetization reversal and electromagnetic radiation due to collective LandauZener relaxation in a crystal of molecular magnets. Analytical and numerical solutions for the time dependence of the relaxation process are obtained. The power of the radiation and the total emitted energy are computed as functions of the crystal parameters and the field sweep rate.
\end{abstract}

PACS numbers: $75.50 . \mathrm{Xx}, 42.50 . \mathrm{Fx}$

\section{INTRODUCTION}

Paramagnetic crystals of high-spin molecular magnets, like $\mathrm{Mn}_{12}, \mathrm{Fe}_{8}$ and others, exhibit unusual magnetic properties related to the macroscopic time of the transition between spin-up and spin-down states of individual magnetic molecules [1]. The latter is due to the high magnetic anisotropy and a large value of spin, $S \gg 1$. For, e.g., a biaxial molecule ( $\mathrm{Fe}_{8}$ of $\left.S=10\right)$, in the magnetic field, $\mathbf{H}$, parallel to the anisotropy axis $Z$, the spin Hamiltonian is

$$
\mathcal{H}=-D S_{z}^{2}+A S_{x}^{2}-g \mu_{B} H_{z} S_{z}
$$

where $g$ is the gyromagnetic factor, $\mu_{B}$ is the Bohr magneton, and $D>A>0$. For small $A$, the approximate energy states of $\mathcal{H}$ are the eigenstates of $S_{z}: S_{z}|m\rangle=$ $m|m\rangle$. At $H_{z}=k D / g \mu_{B}$, with $k=0, \pm 1, \pm 2, \ldots$, the levels $m<0$ and $m^{\prime}$ satisfying $m+m^{\prime}=-k$ come to resonance. For the even $S$, the tunnel splitting of the resonant levels, $\Delta_{m}$, appears (for even $k$ ) in the $\left[\left(m^{\prime}-m\right) / 2\right]$-th order of the perturbation theory on $A$ : $\Delta_{(m=-S)} \propto(A / D)^{\left(m^{\prime}-m\right) / 2}$. At, e.g., $k=0$ (see Fig. (1), $\Delta_{(m=-S)} \propto(A / D)^{S}$ and, thus, at $S=10$, the probability of the transition between spin-up and spin-down states is low. Consequently, at low temperature, the crystal can be prepared in a state with inverse population of the spin energy levels, e.g., magnetized against the direction of the magnetic field. This allows one to observe, in a macroscopic experiment, such quantum effects as resonant spin tunneling 2, 3], spin Berry phase [4], crossover between quantum tunneling and thermal activation [5, 6, 7], and quantum selection rules in the absorption of electromagnetic radiation [8].

Recently, it has been suggested 9, 10, 11] that a crystal of molecular nanomagnets can be a source of coherent electromagnetic radiation in the millimeter wavelength range, highly desirable for applications 12 . Some experimental evidence of this effect has been obtained 10, 13, 14]. The effect is related to Dicke superradiance 15. . Normally, atoms or molecules of a gas, initially prepared in the excited energy state, decay independently by spontaneous emission of light. The power of the radiation obeys the law $P \propto N \exp (-t / \tau)$ where $N$ is the total number of atoms and $\tau$ is the lifetime of the excited state. Dicke argued that $N$ atoms confined within a vol-

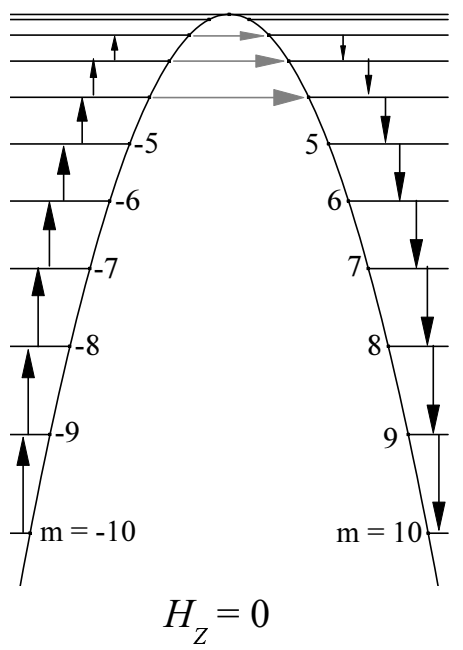

FIG. 1: Approximate energy levels of a spin-10 molecule in a zero magnetic field. The tunnel splitting of the degenerate levels is not shown. Arrows show the relaxation path from $m=-10$ to $m=10$ through thermally assisted quantum tunneling.

ume of size $d$, which is small compared to the wavelength of the radiation $\lambda$, cannot radiate independently from each other. At $d<\lambda$ a spontaneous phase locking of the atomic dipoles takes place, that results in the coherent radiation burst of power $P_{S R} \propto N^{2}$, emitted within a time of order $\tau_{S R} \sim \tau / N$. This phenomenon, called superfluorescence, has been widely observed in gases. It can occur in any system of identical quantum objects if the system is not very large compared to the wavelength of the radiation [16. For crystals of molecular magnets this is true for both, the transitions between the tunnel-split levels, Fig. 2 and the transitions between the adjacent $|m\rangle$ levels, Fig. [1]

In a typical experiment, one magnetizes the crystal and then sweeps the field in the opposite direction. In this paper we will be concerned with the situation when the electromagnetic transitions occur between tunnel-split levels, Fig. [2] These can be, e.g., transitions between $m=-10$ and $m=10$ levels shown in Fig. 1 The electromagnetic relaxation of that kind corresponds to the total magnetization reversal accompanied by the broad band superradiance. It is described by a rigorous model [9] 


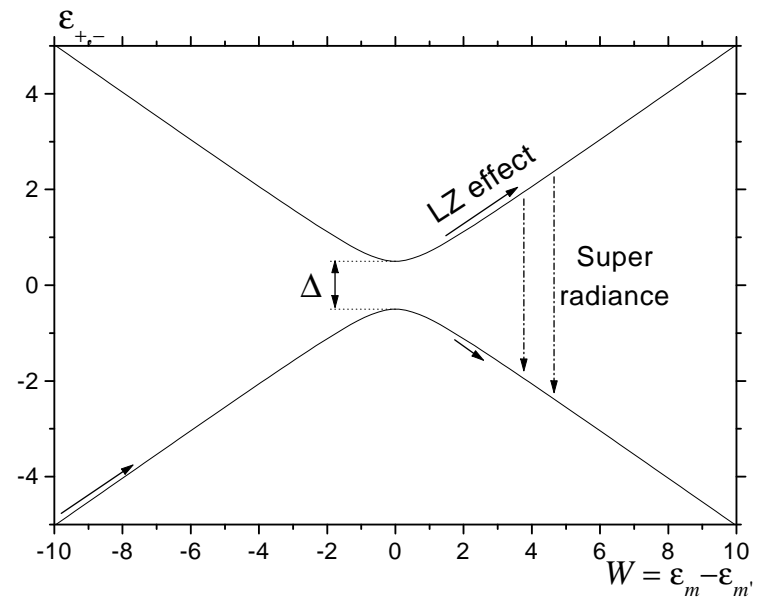

FIG. 2: A pair of tunnel-split levels vs. energy bias $W$. The Landau-Zener (LZ) transition is followed by the emission of the coherent light via superradiance.

which is reviewed in Section II. In essence, if one neglects the electromagnetic radiation, the crossing of the $\left(m, m^{\prime}\right)$ resonance by the magnetic field sweep, Fig. [2] is described by the Landau-Zener theory [17]. When the coupling between the spins and the electromagnetic radiation is taken into account, the magnetic state resulting from the LZ transition relaxes towards the lowest energy state via superradiance. The rate of the superradiant decay, as well as the time dependence of the relaxation, are sensitive to the parameters of the crystal and to the shape of the magnetic field pulse. Our goal is to compute the time dependence of the radiation power and the total radiated energy as functions of the field-sweep rate, the tunnel splitting, and the size of the crystal. This is done in Section III by analytical and numerical methods. Practical implications of our findings are discussed in Section IV.

\section{COLLECTIVE LANDAU-ZENER RELAXATION}

Consider a crystal of $N$ magnetic molecules occupying an $m$ magnetic state that is close to the resonance with the $m^{\prime}$ state, e.g. $m=-S, m^{\prime}=S$ in Fig. [1] We shall assume that the molecules weakly interact with each other through the electromagnetic field. As has been shown in Ref. 9], the quantum magnetic relaxation of such a crystal satisfies the Landau-Lifshitz equation:

$$
\dot{\mathbf{n}}=\gamma\left[\mathbf{n} \times \mathbf{H}_{\mathrm{eff}}\right]-\alpha \gamma\left[\mathbf{n} \times\left[\mathbf{n} \times \mathbf{H}_{\mathrm{eff}}\right]\right] .
$$

Here $\mathbf{n}$ is a unit vector of the pseudospin describing the two-state system, such that $n_{z}=-1$ corresponds to all molecules in the $m$-state, while $n_{z}=1$ corresponds to all molecules in the $m^{\prime}$-state, $\gamma=g \mu_{B} / \hbar$ is the gyromagnetic ratio, the effective magnetic field is given by

$$
g \mu_{B} \mathbf{H}_{\mathrm{eff}}=\Delta \mathbf{e}_{x}+W \mathbf{e}_{z},
$$

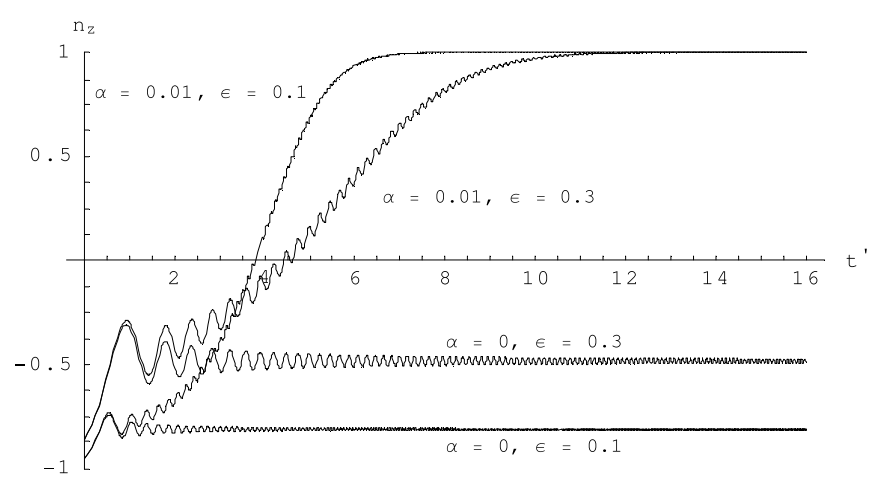

FIG. 3: Time dependence of the magnetization reversal for two values of $\epsilon$ due to pure Landau-Zener relaxation of individual molecules $(\alpha=0)$ and due to collective relaxation via superradiance $(\alpha=0.01)$.

with $\dot{W}(t)=\frac{1}{2} g \mu_{B}\left(m^{\prime}-m\right) \dot{H}_{z}$ being the energy sweep rate (see Fig. (2), and $\alpha \ll 1$ is a dimensionless effective damping coefficient,

$$
\alpha=\frac{1}{24} N\left(m^{\prime}-m\right)^{2} g^{2} \alpha_{o}\left(\frac{\Delta}{m_{e} c^{2}}\right)^{2},
$$

with $\alpha_{o}=e^{2} / \hbar c \approx 1 / 137$ being the fine structure constant. Note that $\alpha$ is independent of the magnetic field.

The first term in Eq. (2) gives dissipationless LandauZener transitions when the field is swept through the resonance such that $W=W(t)$ satisfies $W( \pm \infty)= \pm \infty$, and the initial condition is $\mathbf{n}(-\infty)=-\mathbf{e}_{z}$. Indeed, at $\alpha=0$ the Schrödinger equation for a two-level system is equivalent to the equation for a precessing spin. The probability $p(t)$ for the molecule to stay in the initial state is given by

$$
p(t)=\left[1-n_{z}(t)\right] / 2 .
$$

For $W(t)=v t$, one obtains the famous Landau-Zener result [17]: $p(\infty) \equiv p_{L Z}=\exp (-\epsilon)$, where

$$
\epsilon=\frac{\pi \Delta^{2}}{2 \hbar v} \text {. }
$$

The Landau-Zener effect corresponds to only partial magnetization reversal,

$$
n_{z}^{L Z}(\infty)=1-2 \exp (-\epsilon),
$$

see Fig. 3 at $\alpha=0$. The value of $n_{z}^{L Z}(\infty)$ is close to -1 at $\epsilon \ll 1$, that is for the fast field sweep. In this case most of the molecules, after crossing the resonance, remain in the initial $m$-state by passing from the lower to the upper branch in Fig. 2 On the contrary, for a slow sweep, that is when $\epsilon \gg 1$, most of the molecules follow the lower branch in Fig. 2 and the final state of the crystal is exponentially close to $n_{z}^{L Z}(\infty)=1$.

The second term in Eq. (2) describes collective magnetic relaxation via Dicke superradiance. Due to this term the magnetization of the entire crystal at long times 
reverses completely to $n_{z}(\infty)=1$, as is shown in Fig. [3 for a finite $\alpha$. The collective relaxation due to superradiance is significant for $\epsilon \lesssim 1$, that is, when $n_{z}^{L Z}(\infty)$ is not very close to 1 . Thus, the observation of the superradiance requires a fast field sweep.

\section{RADIATION POWER}

The power of the superradiance described by Eq. (2) can be obtained from the classical formula for the magnetic dipole radiation [9]:

$$
P(t)=\left[2 /\left(3 c^{3}\right)\right] \ddot{m}_{z}^{2}(t),
$$

where

$$
m_{z}(t)=\frac{1}{2} N\left(m-m^{\prime}\right) g \mu_{B} n_{z}(t) .
$$

Close to the $\left(m, m^{\prime}\right)$ resonance, nearly any field sweep of practical interest is linear in time, $W=v t$. It is convenient to use dimensionless variables:

$$
t^{\prime}=\frac{t \Delta}{\hbar}, \quad W^{\prime}\left(t^{\prime}\right)=\frac{v t}{\Delta}=\frac{\hbar v t^{\prime}}{\Delta^{2}}=\frac{\pi t^{\prime}}{2 \epsilon} .
$$

In terms of these variables Eq. (2) and Eq. (8) become

$$
\frac{d \mathbf{n}}{d t^{\prime}}=\left[\mathbf{n} \times\left(\mathbf{e}_{x}+W^{\prime}\left(t^{\prime}\right) \mathbf{e}_{z}\right)\right]-\alpha\left[\mathbf{n} \times\left[\mathbf{n} \times\left(\mathbf{e}_{x}+W^{\prime}\left(t^{\prime}\right) \mathbf{e}_{z}\right)\right]\right]
$$

and

$$
P=\alpha N \hbar^{-1} \Delta^{2}\left(\frac{d^{2} n_{z}}{d t^{\prime 2}}\right)^{2}
$$

The total emitted energy, $E=\int d t P(t)$, is given by

$$
E=\alpha N \Delta E^{\prime},
$$

where we have introduced dimensionless

$$
E^{\prime}=\int d t^{\prime}\left(\frac{d^{2} n_{z}}{d t^{\prime 2}}\right)^{2}
$$

\section{A. Analytical}

We shall start by developing an analytical approximation for the practical case of $\epsilon<1$ and $\alpha \ll 1$. The time interval of interest is the one past the Landau-Zener transition: $W^{\prime} \gg 1$. In this case, retaining the leading terms in Eq. (11), we get

$$
\begin{aligned}
& \frac{d n_{x}}{d t^{\prime}}=W^{\prime} n_{y} \\
& \frac{d n_{y}}{d t^{\prime}}=-W^{\prime} n_{x} \\
& \frac{d n_{z}}{d t^{\prime}}=-n_{y}+\alpha W^{\prime}\left(t^{\prime}\right)\left(1-n_{z}^{2}\right) .
\end{aligned}
$$

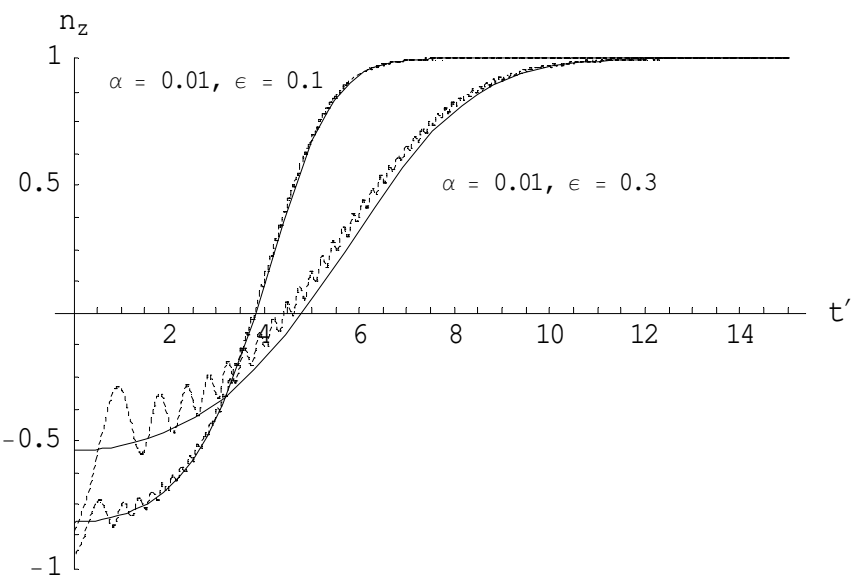

FIG. 4: Approximate analytical solution for $n_{z}\left(t^{\prime}\right)$ averaged over oscillations, Eq. (20), for two values of $\epsilon$ and $\alpha=0.01$ (solid line). The numerical solution of Eq. (11) is shown by the dash line.

These equations show that $n_{x}$ and $n_{y}$ oscillate rapidly in time, while $n_{z}$, in accordance with Fig. 3 has a slowly varying average. Averaging Eq. (17) over the period of oscillations of $n_{y}$, one obtains:

$$
\frac{d \bar{n}_{z}}{d t^{\prime}}=\alpha W^{\prime}\left(t^{\prime}\right)\left(1-\bar{n}_{z}^{2}\right) .
$$

Eq. (18) describes the superradiant stage of the evolution of $\bar{n}_{z}$. Therefore, it must be solved with the initial condition $\bar{n}_{z}=n_{z}^{L Z}$ at $t=0$. At small $\epsilon$, Eq. (7) gives for that initial condition:

$$
\bar{n}_{z}(0)=-1+2 \epsilon .
$$

The corresponding solution of Eq. (18) reads 14]

$$
\bar{n}_{z}\left(t^{\prime}\right)=\tanh \left(\frac{\alpha \pi t^{\prime 2}}{4 \epsilon}-\frac{1}{2} \ln \frac{1}{\epsilon}\right) .
$$

It is shown by the solid line in Fig. (4 For $\epsilon=0.1$ Eq. (18) is, clearly, a good approximation to the full solution averaged over oscillations. As $\epsilon$ increases, some discrepancy is observed. One can improve the analytical approximation by writing $n_{z}=\bar{n}_{z}+\delta n_{z}$ and solving Eq. (17) through iterations, but this, at the end, will require a numerical integration, so that the improvement obtained by this method does not give much advantage over the direct numerical solution of Eq. (11).

The approximate solution of Eqs. (15) and (16), satisfying $\mathbf{n}^{2}=1$, is given by

$$
\begin{aligned}
& n_{x}=\sqrt{1-\bar{n}_{z}^{2}} \sin \left(\frac{\pi t^{\prime 2}}{4 \epsilon}+\phi_{0}\right) \\
& n_{y}=-\sqrt{1-\bar{n}_{z}^{2}} \cos \left(\frac{\pi t^{\prime 2}}{4 \epsilon}+\phi_{0}\right),
\end{aligned}
$$




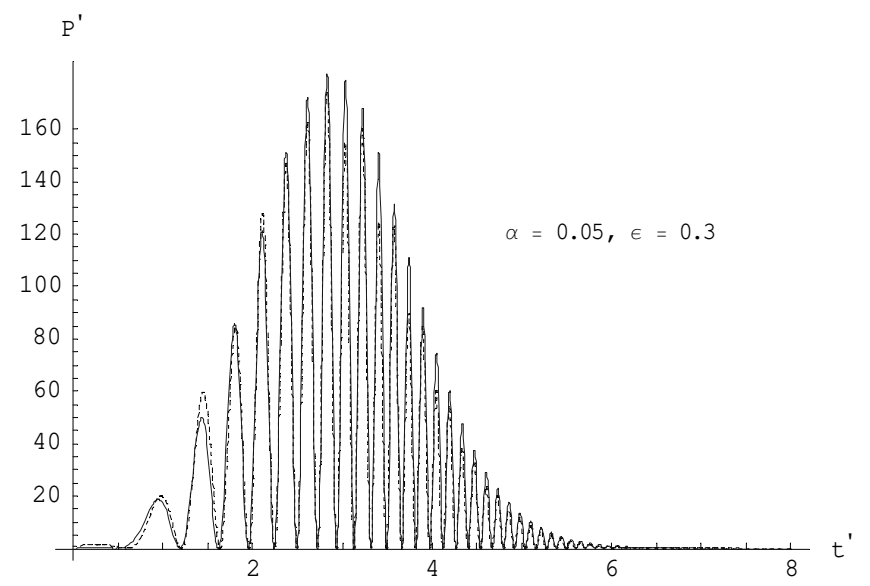

FIG. 5: Time dependence of the reduced radiation power, $P^{\prime}=\left(d^{2} n_{z} / d t^{\prime 2}\right)^{2}$ at $\epsilon=0.3$ and $\alpha=0.05$. Solid line represents numerical results. Dash line corresponds to Eq. (22) at $\phi_{0}=2.576$.

where $\phi_{0}$ is a phase which we are not attempting to compute analytically. One can see from Eqs. (20) and (21) that $\bar{n}_{z}$, indeed, changes slowly with time, compared to the oscillations of $n_{x}\left(t^{\prime}\right)$ and $n_{y}\left(t^{\prime}\right)$, because of the condition $\alpha \ll 1$.

Let us now turn to the analytical approximation for the power and the total radiated energy. It is easy to see from Eqs. (17)- 21) that the main contribution to $d^{2} n_{z} / d t^{\prime 2}$ is determined by the rapidly oscillating $n_{y}$-term in the right-hand-side of Eq. (17):

$$
\frac{d^{2} n_{z}}{d t^{\prime 2}}=-\left(\frac{\pi t^{\prime}}{2 \epsilon}\right) \frac{\sin \left[\left(\pi t^{\prime 2} / 4 \epsilon\right)+\phi_{0}\right]}{\cosh \left[\left(\alpha \pi t^{\prime 2} / 4 \epsilon\right)+\frac{1}{2} \ln \epsilon\right]}
$$

Substituting this expression into Eq. (14) and replacing the rapidly oscillating $\sin ^{2}\left(\pi t^{\prime 2} / 4 \epsilon\right)$ under the integral by $1 / 2$, one finally obtains:

$$
E^{\prime}=\frac{\sqrt{\pi}}{\epsilon^{1 / 2} \alpha^{3 / 2}} \int_{0}^{\infty} \frac{x^{2} d x}{\cosh ^{2}\left(x^{2}+\frac{1}{2} \ln \epsilon\right)} .
$$

Equations (12), (13), (14), (22), and (23) give the dependence of the radiation power and the total emitted energy on the field sweep rate and on the parameters of the crystal.

\section{B. Numerical}

We shall now compute $P(t)$ and $E$ by numerical integration of Eq. (11), and compare them with our analytical findings.

The time dependence of the reduced power, $P^{\prime}=$ $\left(d^{2} n_{z} / d t^{\prime 2}\right)^{2}$, is shown in Fig. 5] The comparison with Eq. (22) is performed by fitting the value of $\phi_{0}$ until the match with the numerical solution of Eq. (11) for $\left(d^{2} n_{z} / d t^{\prime 2}\right)^{2}$ is obtained. Even for $\epsilon$ as large as 0.3 the

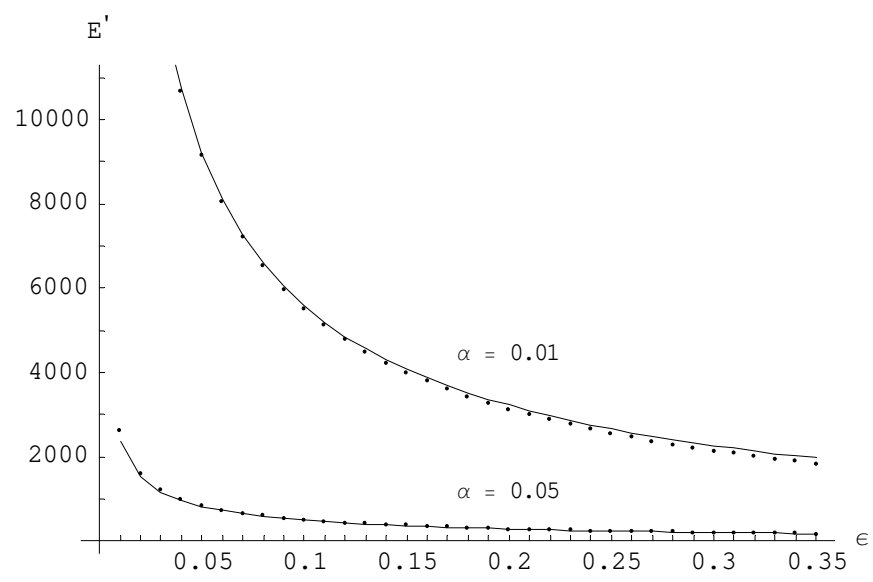

FIG. 6: The $\epsilon$ dependence of the total emitted energy at two values of $\alpha$. Points represent numerical results. Solid line corresponds to Eq. 23.

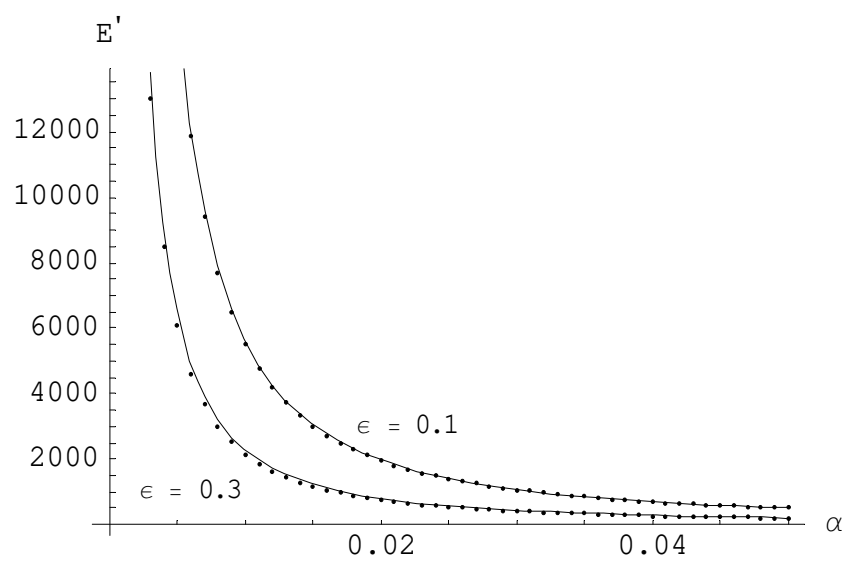

FIG. 7: The $\alpha$ dependence of $E^{\prime}$ at two values of $\epsilon$. Points represent numerical results. Solid line corresponds to Eq. (23).

agreement of the numerical results with the analytical formula is rather good. Note that the oscillation of the power in time is a quantum effect related to the oscillation of $n_{z}$.

Fig. 6] shows the dependence of the total emitted energy on the parameter $\epsilon$, that is, on the inverse field sweep rate. Fig. 7 shows the dependence of $E^{\prime}$ on the parameter $\alpha$.

The question of significant importance for experiment is the spectral composition of the radiation. The total emitted energy can be presented as

$$
E=\int d \omega I(\omega)
$$

where

$$
I(\omega)=\hbar \alpha N I^{\prime}\left(\omega^{\prime}\right)
$$




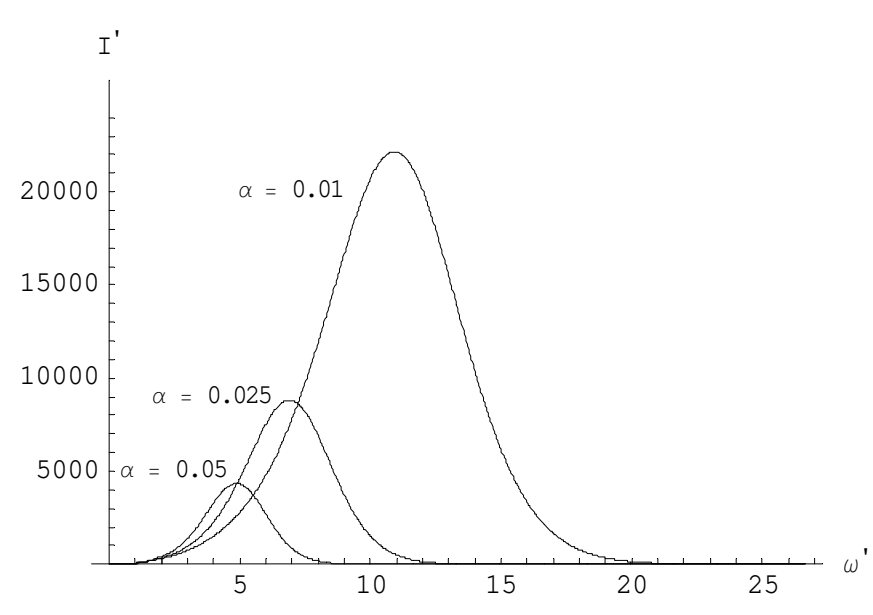

FIG. 8: Spectral function $I^{\prime}\left(\omega^{\prime}\right)$ for three values of alpha at $\epsilon=0.1$.

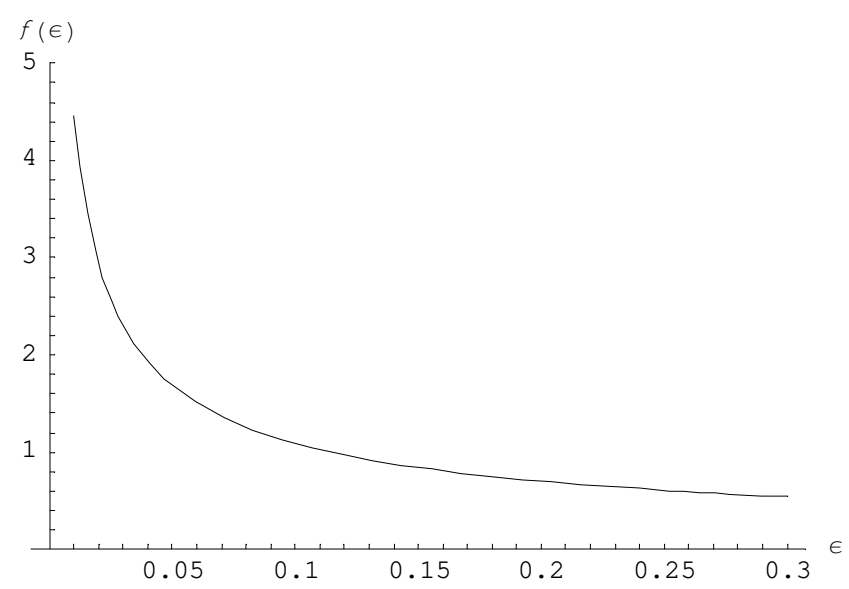

FIG. 9: Dependence of $f$ of Eq. (27) on $\epsilon$.

is the spectral power. Here $I^{\prime}\left(\omega^{\prime}\right)$ is a dimensionless function of the dimensionless frequency, $\omega^{\prime}=\hbar \omega / \Delta$. It must be computed via the Fourier transform of $d^{2} n_{z} / d t^{\prime 2}$ :

$$
I^{\prime}\left(\omega^{\prime}\right)=\frac{1}{2 \pi}\left|\int d t^{\prime} e^{i \omega^{\prime} t^{\prime}}\left(\frac{d^{2} n_{z}}{d t^{\prime 2}}\right)\right|^{2}
$$

This function is shown in Fig. 8 The peak of the power occurs at

$$
\hbar \omega_{\max }=\frac{\Delta}{\sqrt{\alpha}} f(\epsilon)
$$

This scaling of $\hbar \omega_{\max }$ on $\alpha$ follows from Eq. (11). The function $f(\epsilon)$, computed numerically, is shown in Fig. 9

\section{DISCUSSION}

The formulas and the numerical results obtained above are valid if the energy distance between the resonant levels, $W$, is small compared to the distance between the adjacent $m$-levels. For numerical estimates, we shall stick to the $(-S, S)$-resonance. The conclusions of this section, however, will apply to other resonances as well. For the model illustrated by Eq. (1) and Fig. 11 the distance between the $m=-S$ level and the $m=-S+1$ level is $(2 S-1) D \approx 2 S D$. The validity condition we are looking for is then $W(t) \ll 2 S D$. For $W=v t$ one should verify this condition at $W_{\max }=v t_{\max }$, where $t_{\max }=\hbar t_{\text {max }}^{\prime} / \Delta$ is the time when the superradiance drops exponentially due to the hyperbolic cosine in Eq. (22). According to this equation and Eq. (10), $t^{\prime}{ }_{\max } \sim \sqrt{\epsilon / \alpha}$ and $W_{\max } \sim \Delta / \sqrt{\epsilon \alpha}$. Substituting this into $W_{\max } \ll 2 S D$ and using Eq. (4) for $\alpha$, one obtains the validity condition in the form of the lower bound on the total number of molecules:

$$
N \gg \frac{1}{\epsilon \alpha_{o}}\left(\frac{m_{e} c^{2}}{U_{a}}\right)^{2},
$$

where $U_{a}=D S^{2}$ is the energy barrier between $m= \pm S$ states due to magnetic anisotropy. Eq. (28) shows that a high magnetic anisotropy and a not very small $\epsilon$ are needed if the size of the system is to remain within reasonable limits. The optimal would be $\epsilon \sim 1$ since, according to Eq. (7), $\epsilon \lesssim 1$ (that is, a sufficiently high field-sweep rate) is needed to create an inverse population of spin levels. For $\mathrm{Mn}_{12}$ and $\mathrm{Fe}_{8}$, the anisotropy barrier is of order $60 \mathrm{~K}$ and $30 \mathrm{~K}$ respectively, and the lower bound on $N$, according to Eq. (28), must be between $10^{18}$ and $10^{19}$ molecules. With account of the unit cell volume $\left(3.7 \mathrm{~nm}^{3}\right.$ and $2.0 \mathrm{~nm}^{3}$ for $\mathrm{Mn}_{12}$ and $\mathrm{Fe}_{8}$, respectively) this translates into a volume of order or greater than $1 \mathrm{~mm}^{3}$. Remarkably, this agrees with the reported lower bound on the volume of the crystal (or crystal assembly) that shows evidence of electromagnetic radiation during magnetization reversal [10, 13, 14].

We shall now estimate the total emitted energy and the power of the radiation. According to Eq. (23), $E^{\prime} \sim$ $\epsilon^{-1 / 2} \alpha^{-3 / 2}$. This gives for $E$ of Eq. (13): $E \sim N \Delta / \sqrt{\epsilon \alpha}$. With the help of Eq. (4) one obtains:

$$
E \sim \epsilon^{-1 / 2} N^{1 / 2} m_{e} c^{2} .
$$

For the purpose of the order-of-magnitude estimate we have dropped the factor $g S \sqrt{\alpha_{0}}$ of order unity. Notice that the total emitted energy is proportional to the square root of the crystal volume. At $\epsilon \sim 1$ and $N \sim 10^{18}$, Eq. (29) provides $E \sim 0.1 \mathrm{~mJ}$.

According to Eqs. (12) and (22) (see also Fig. (5) the power of the radiation oscillates in time. In most cases, observation of these oscillations must be impeded by the finite time resolution of the measuring equipment, so that only the envelope of the curve shown in Fig. 5 will be observed. The peak power can be estimated as $P_{\max } \sim$ 
$E / t_{\max } \sim N \Delta^{2} / \hbar \epsilon$. Substituting here $\epsilon$ of Eq. (6), one obtains

$$
P_{\max } \sim N v \sim \delta M \frac{d H}{d t},
$$

where we have introduced $\delta M=g \mu_{B}\left(m^{\prime}-m\right) N$, the change in the total magnetic moment of the crystal due to collective electromagnetic relaxation. Note that the relations $E \propto \sqrt{N}$ and $P_{\max } \propto N$ are specific to the radiation problem we have studied. For an assembly of a few mm-size crystals Eq. (30) gives $P_{\max } \sim 10 \mu \mathrm{W}$ at a typical laboratory field sweep rate of $0.01 \mathrm{~T} / \mathrm{s}$ [10, 13] and $P_{\max } \sim 1 \mathrm{~W}$ for a fast field pulse, $d H / d t \sim 10^{3} \mathrm{~T} / \mathrm{s}$ 14]. Note, however, that in the case of an ultrafast sweep the condition $\epsilon \sim 1$ can be satisfied only by a large tunnel splitting $\Delta$, making the preparation of the initially magnetized state less simple than in the case of small $\Delta$.

During the adiabatic sweep, the frequency of the radiation is determined by the distance between the spin levels, $\hbar \omega=\sqrt{\Delta^{2}+W^{2}}$. For the most of the relaxation process, $W \gg \Delta$, and, thus, $\omega=W(t) / \hbar$. The peak of the spectral power, Fig. [8] corresponds to $\hbar \omega_{\max } \sim W\left(t_{\max }\right) \sim \Delta / \sqrt{\epsilon \alpha}$. Up to a factor of order unity, that depends logarithmically on $\epsilon$, this coincides with Eq. (27). The logarithmic difference of $f(\epsilon)$ in Fig. 9 from $1 / \sqrt{\epsilon}$ is due to $\ln \epsilon$ in Eq. (22). With the help of Eq. (4), we obtain that by order of magnitude

$$
\omega_{\max } \sim \frac{m_{e} c^{2}}{\hbar \sqrt{\epsilon N}},
$$

where we again omitted the factor $g S \sqrt{\alpha_{0}}$ of order unity. For $\epsilon \sim 1$ and $N \sim 10^{18}$, required to produce significant radiation (see above), this frequency is in the terahertz range.

For the radiation to be coherent, the inhomogeneous broadening of $\epsilon$ must be small throughout the crystal.
This translates into narrow distribution of the tunnel splitting and narrow distribution of the magnetic field felt by the spins. Both conditions must be satisfied in $\mathrm{Fe}_{8}$ because they are also the necessary conditions for the Berry phase effect observed in that system [4]. In $\mathrm{Mn}_{12}$ the situation is less clear due to solvent disorder, large hyperfine interactions, dislocations, etc., which result in a distribution of $\Delta[18,19,20,21,22]$. The suitability of $\mathrm{Mn}_{12}$ for the study of superradiance depends on whether the distribution of $\Delta$ is continuous or consists of a finite number of narrow lines due to, e.g., finite number of nuclear spin states, presence of isomers in the structure of the molecule [20], etc. Systems with narrow distribution of $\Delta$ probably exist among hundreds of new molecular magnets synthesized in recent years. In such systems, narrow distribution of the magnetic field should be achieved automatically when the spins of the initially saturated sample rotate coherently due to superradiance.

\section{CONCLUSIONS}

We have studied magnetic relaxation via coherent electromagnetic radiation, produced by the magnetic field sweep in a crystal of molecular magnets on crossing the tunneling resonance. We find that the effect exists starting roughly with crystals (or crystal assembly) of millimeter size. The radiation is broadband with the cutoff in the terahertz range. The power of the radiation is proportional to the field-sweep rate and ranges from microwatts to watts for the existing sweep rates.

\section{ACKNOWLEDGMENTS}

This work has been supported by the NSF Grant No. EIA-0310517.
[1] R. Sessoli, D. Gatteschi, A. Ganeschi, and M. A. Novak, Nature (London) 365, 141 (1993).

[2] J. R. Friedman, M. P. Sarachik, J. Tejada, and R. Ziolo, Phys. Rev. Lett. 76, 3830 (1996).

[3] J. M. Hernandez, X. X. Zhang, F. Luis, J. Bartolomé, J. Tejada, and R. Ziolo, Europhys. Lett. 35, 301 (1996); L. Thomas, F. Lionti, R. Ballou, D. Gatteschi, R. Sessoli, and B. Barbara, Nature (London) 383, 145 (1996); C. Sangregorio, T. Ohm, C. Paulsen, R. Sessoli, and D. Gatteschi, Phys. Rev. Lett. 78, 4645 (1997).

[4] W. Wernsdorfer and R. Sessoli, Science 284, 133 (1999).

[5] E. M. Chudnovsky and D. A. Garanin, Phys. Rev. Lett. 79, 4469 (1997); D. A. Garanin and E. M. Chudnovsky, Phys. Rev. B56, 11102 (1997).

[6] A. D. Kent, Y. Zhong, L Bokacheva, D. Ruiz, D. N. Henrickson, and M. P. Sarachik, Europhys. Lett. 49, 521 (2000).

[7] L. Bokacheva, A. D. Kent, and M. A. Walters, Phys. Rev. Lett. 85, 4803 (2000); K. M. Mertes, Y. Suzuki,
M. P. Sarachik, Y. Paltiel, H. Shtrikman, E. Zeldov, E. Rumberger, and D. N. Hendrickson, Europhys. Lett. 55, 874 (2001).

[8] L. Sorace, W. Wernsdorfer, C. Thirion, A.-L. Barra, M. Pacchioni, D. Mailly, and B. Barbara, Phys. Rev. B68, 220407 (2003).

[9] E. M. Chudnovsky and D. A. Garanin Phys. Rev. Lett. 89, 157201 (2002).

[10] J. Tejada, R. Amigo, J. M. Hernandez, and E. M. Chudnovsky, Phys. Rev. 68, 014431 (2003).

[11] V. K. Henner and I. V. Kaganov, Phys. Rev. 68, 144420 (2003).

[12] D. Mittleman, Sensing with Terahertz Radiation, Springer (2003).

[13] J. Tejada, E. M. Chudnovsky, J. M. Hernandez, and T. Amigo, Appl. Phys. Lett. 84, 2373 (2004).

[14] J. Vanacken, S. Stroobants, M. Malfait, V. V. Moshchalkov, M. Jordi, J. Tejada, R. Amigo, E. M. Chudnovsky, and D. A. Garanin, 
arXiv:cond-mat/0404041

[15] R. H. Dicke, Phys. Rev. 9399 (1954).

[16] E. M. Chudnovsky and D. A. Garanin, arXiv:cond-mat/0404239

[17] L. D. Landau, Phys. Z. Sowjetunion 2, 46 (1932); C. Zener, Proc. R. Soc. London, Ser. A, 137, 696 (1932).

[18] E. M. Chudnovsky and D. A. Garanin, Phys. Rev. Lett. 87, 187203 (2001); D. A. Garanin and E. M. Chudnovsky, Phys. Rev. B65, 094423 (2002).

[19] K. M. Mertes, Y. Suzuki, M. P. Sarachik, Y. Paltiel, H. Shtrikman, E. Zeldov, E. Rumberger, D. N. Hendrickson, and G. Christou, Phys. Rev. Lett. 87, 227205 (2001)

[20] A. Cornia, R. Sessoli, L. Sorace, D. Gatteschi, and A. L. Barra, Phys. Rev. Lett. 89, 257201 (2002).

[21] S. Hill, R. S. Edwards, S. I. Jones, J. M. North, and N. S. Dalal, Phys. Rev. Lett. 90, 217204 (2003).

[22] E. del Barco, A. D. Kent, E. M. Rumberger, D. N. Hendrickson, and G. Christou, Phys. Rev. Lett. 91, 047203 (2003); E. del Barco, A. D. Kent, N. E. Chakov, L. N. Zakharov, A. L. Rheingold, D. N. Hendrickson, and G. Christou, Phys. Rev. 69, 020411(R) (2004). 